\title{
Upland rice root parameters and their relationship on utilizing different levels of applied zinc.
}

\begin{abstract}
Global efforts are under way to improve the $\mathrm{Zn}$ concentrations in rice to increase $\mathrm{Zn}$ in diets. Zinc uptake in relation to morphological root parameters among 7 upland rice varieties was studied by conducting a solution culture experiment using modified Yoshida solution in Agriculture Faculty of University Putra Malaysia. Five zinc levels were developed by the addition of $0,5,10,20,30 \mathrm{mgL}-1 \mathrm{ZnSO}$. Seedlings were harvested in week 4 . Zn uptake in roots of upland rice showed significant differences among all varieties. Zn uptake significantly increased at all rates. Other root parameters (length, average diameter, surface area, volume, and number of root tips) did not show any significant differences in 0 to $20 \mathrm{mg}$ $\mathrm{ZnL}-1$, but they decreased significantly in $30 \mathrm{mg} \mathrm{ZnL-1} \mathrm{in} \mathrm{4th} \mathrm{week} \mathrm{of} \mathrm{observation.}$
\end{abstract}

Keyword: Zinc; Rice; Root morphology; Zn uptake. 